\title{
Mass Transfer Assessment in Extraction of La(III) and Ce(III) Ions Using Rotating Scheibel Column with Regular Packing
}

\author{
Mehdi Asadollahzadeh ${ }^{1}$, Rezvan Torkaman ${ }^{2}$, Meisam Torab-Mostaedi $^{2}$, and Mojtaba \\ Saremi $^{2}$ \\ ${ }^{1}$ Iran University of Science and Technology (IUST) \\ ${ }^{2}$ Affiliation not available
}

November 29, 2020

\begin{abstract}
In this study, the Scheibel column with the regular packing structure was investigated in the extraction process of lanthanum and cerium ions. Mass transfer coefficients identified the column performance in the transport stage of La(III) and Ce(III) from the aqueous phase to the organic phase. The variations in operating conditions were investigated to optimize the extraction of ions. The backflow model (BFM), axial dispersion model (ADM), and forward mixing model (FMM) were investigated to evaluate the mass transfer performance. The result showed that the description of the volumetric overall mass transfer coefficient with forward mixing model is more significant than two other models. The medium of average absolute relative error obtained by the FMM model is $\sim 4.45 \%$ and $\sim 18.40 \%$ (lanthanum extraction) and $\sim 10.75 \%$ and $\sim 14.99 \%$ (cerium extraction) for continuous and dispersed phase, respectively. It indicated that the applied model has a remarkable accuracy for description of mass transfer performance
\end{abstract}

\section{Hosted file}

Manuscript_AIChE.pdf available at https://authorea.com/users/379778/articles/495989-masstransfer-assessment-in-extraction-of-la-iii-and-ce-iii-ions-using-rotating-scheibelcolumn-with-regular-packing 